\title{
Assessing Confidence in Detections of Extraterrestrial Life
}

\author{
CATHARINE A. CONLEY ${ }^{1,3}$ AND ANDREW STEELE ${ }^{2}$
}

${ }^{1}$ NASA Ames Research Center, Moffett Field, CA

${ }^{2}$ Carnegie Institution for Science, Geophysical Lab, Washington, DC

${ }^{3}$ To whom correspondence should be addressed

Humans have speculated about the existence of life on other planets for centuries. The invention of the telescope was rapidly followed by claims reporting remote observation of indigenous biosignatures on the Moon and Mars, and meteorites collected on Earth were also reported to contain indigenous biological material -- however, all these claims have justifiably been considered to have very low statistical confidence. More recently, the Viking landers on Mars reported detecting simple organic compounds that were initially interpreted as Earth contamination, and only recognized as indigenous to Mars after results from subsequent missions prompted re-evaluation of the raw data from Viking. This work represents initial steps in developing mathematically-rigorous approaches to assessing the level of statistical confidence appropriate to any claimed detection of extraterrestrial life. The establishment of decision frameworks, that identify both potential false negative as well as false positive results, will facilitate the ability to recognize correctly when indigenous biology, abiotic compounds, and/or contamination from modern Earth are present. 